\title{
Micro(RNA) management and mismanagement of the islet
}

\author{
Lena Eliasson ${ }^{1}$ and Romano Regazzi ${ }^{2}$
}

1. Islet Cell Exocytosis, Lund University Diabetes Centre, Department of Clinical Sciences Malmö, Lund University, Clinical Research Centre, SUS, Malmö, Sweden

2. Department of Fundamental Neurosciences, University of Lausanne, Lausanne, Switzerland

Corresponding authors:

Professor Lena Eliasson, Department of Clinical Sciences Malmö, Clinical Research Centre 91-11, Box 50332, 20213 Malmö

Email: lena.eliasson@med.lu.se

Professor Romano Regazzi, Department of Fundamental Neurosciences, Rue du Bugnon 9, 1005 Lausanne, Switzerland.

Email: Romano.Regazzi@unil.ch

Keywords: microRNA, $\beta$-cell, insulin, glucotoxicity, lipotoxicity, therapeutics

Declaration of interest: none 
Graphical abstract

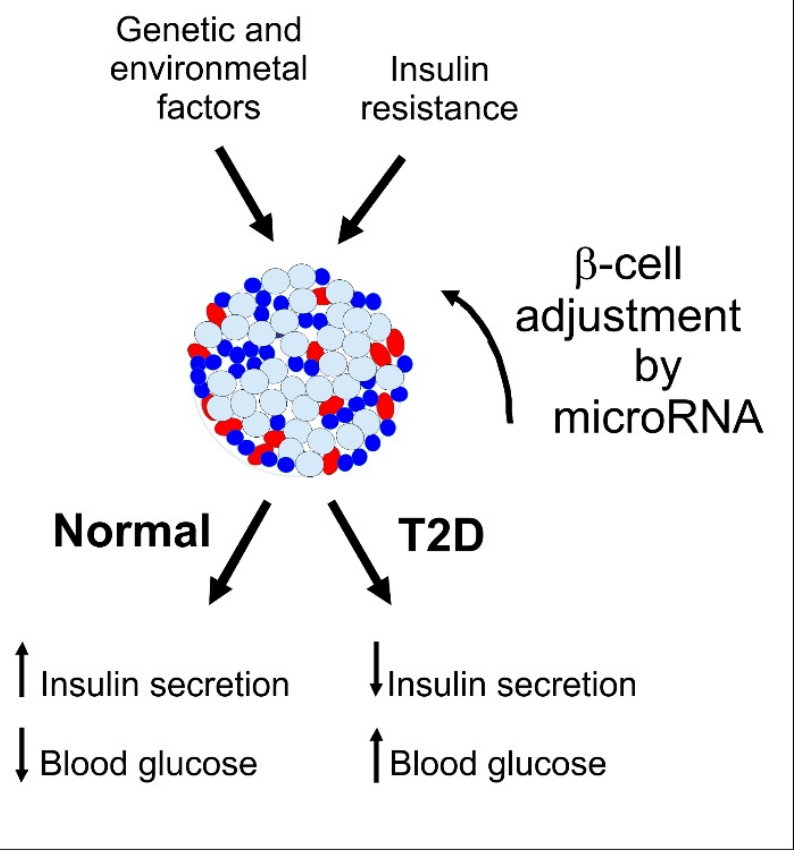




\begin{abstract}
Pancreatic $\beta$-cells located within the islets of Langerhans play a central role in metabolic control. The main function of these cells is to produce and secrete insulin in response to a rise in circulating levels of glucose and other nutrients. The release of insufficient insulin to cover the organism needs results in chronic hyperglycemia and diabetes development. $\beta$-cells insure a highly specialized task and to efficiently accomplish their function they need to express a specific set of genes. MicroRNAs (miRNAs) are small non-coding RNAs and key regulators of gene expression. Indeed, by partially pairing to specific sequences in the $3^{\prime}$ untranslated regions of target mRNAs, each of them can control the translation of hundreds of transcripts. In this review, we focus on few key miRNAs controlling islet function and discuss: their differential expression in Type 2 diabetes (T2D), their regulation by genetic and environmental factors, and their therapeutic potential. Genetic and epigenetic changes or prolonged exposure to hyperglycemia and/or hyperlipidemia can affect the $\beta$-cell miRNA expression profile, resulting in impaired $\beta$-cell function and survival leading to the development of T2D. Experimental approaches permitting to correct the level of mis-expressed miRNAs have been shown to prevent or treat T2D in animal models, suggesting that these small RNAs may become interesting therapeutic targets. However, translation of these experimental findings to the clinics will necessitate the development of innovative strategies allowing safe and specific delivery of compounds modulating the level of the relevant miRNAs to the $\beta$-cells.
\end{abstract}

\title{
Introduction
}

More than ten years have passed since the first publications about the identification of abundant islet microRNAs (miRNAs) [1, 2]. Since then, we have learned much regarding the role played by miRNAs in the post-transcriptional regulation of islet cells (See e.g [3-5]). During the last decades, it also became increasingly clear that failure of insulin-secreting $\beta$-cells plays an important role in the development of Type 2 diabetes (T2D; [6]). The $\beta$-cell is the main cell-type present in the islet of Langerhans and release insulin in response to the increase in blood glucose levels occurring after a meal [7]. T2D is associated with both insulin resistance and impaired insulin secretion. Insulin resistance in peripheral tissues puts an extra demand to the $\beta$-cells and forces them to release more insulin. Moreover, prolonged exposure to elevated levels of lipids and glucose, a condition very often occurring in obese and insulin resistant individuals, perturbs the function of the $\beta$-cells and decrease their survival $[8,9]$. Indeed, defective insulin secretion in T2D is associated with both reduced $\beta$-cell mass and impaired $\beta$-cell function. MiRNAs have been proven to affect both these processes [3-5].

MiRNAs are small non-coding RNAs (21-23 nt) involved in post-transcriptional regulation of gene expression. Each miRNA contains a 6-8 nt long sequence in the 5 '-end called the seed sequence that is important for recognition of the target mRNA. The partial pairing of the seed sequence of each miRNA with the $3^{\prime}$ untranslated regions ( $3^{\prime}$ UTRs), and to some extent the $5^{\prime}$ UTR, of target mRNAs reduces the expression of hundreds of transcripts. Target expression is repressed through translational inhibition and/or mRNA destabilization [10]. The miRNA genes are located within protein-coding genes or are produced from non-coding transcription units in intergenic regions. In most cases, miRNA genes are transcribed in the nucleus by polymerase II into pri-miRNAs either together with their host gene or through the use of their own promoter (for detailed review on miRNA biogenesis see [10]). In some cases, several miRNAs form "clusters" and are produced from a single long transcript [11]. MiRNAs from the same cluster are not necessarily belonging to the same family. Indeed, a family of miRNAs includes all miRNAs that share the same seed sequence, regardless of location in the genome [12]. 
The microprocessor complex containing the DNA-binding protein DGCR8 and the RNase III Drosha[13] recognizes the unique hairpin structure of the pri-miRNAs, which is cleaved to produce the pre-miRNA. Exportin-5 enables the transport of the pre-miRNA out from the nucleus [14]. After exiting the nucleus, the RNase III enzyme Dicer processes the pre-miRNAs to generate mature miRNA duplexes. Knockdown of Dicer specifically in $\beta$-cells leads to diabetes development [15], showing the importance of miRNAs for $\beta$-cell function. The miRNA duplex is then loaded into an Argonaute protein, initiating the formation of the mature silencing complex that will finally contain only one strand (guide strand) of the miRNA duplex while the other (passenger strand) is usually degraded. Once loaded into the silencing complex, the seed sequence of the mature miRNA pairs with the corresponding sites within the target mRNA and represses its translation [10]. Besides seed-dependent recognition of target mRNAs, recent studies $[16,17]$ have suggested the presence of seed-independent targeting of proteincoding sequences (CDS). This class of miRNA recognition elements in the CDS-region pairs with the $3^{\prime}$ end of the miRNA (instead of the $5^{\prime}$ seed) to regulate translation.

The posttranscriptional regulation of gene expression by miRNAs is complex. Often the repression exerted on a specific gene by a single miRNA is modest. However, since full complementarity of the miRNA with the target sequence is not required, each miRNA can control the output of many target genes and each mRNA is often regulated by several miRNAs [12]. The role of miRNAs is to complement and extend the regulatory mechanisms exerted at transcription and by other nuclear events. Thus, the miRNA-mRNA interaction provides an extra layer of post-transcriptional gene regulation. Hence, miRNAs can modify and fine tune the expression of many different cellular pathways, and events interfering with miRNA action may lead to the development of a variety of diseases, including T2D [5, 18].

\section{miRNAs in islet cells}

MiRNA expression analyses have identified miR-375 as one of the most abundant miRNAs in islet cells $[19,20]$. Data from sorted human islet cells suggest that miR-375 is equally expressed in glucagonsecreting $\alpha$-cells as in the insulin-secreting $\beta$-cells [21], however changes in the level of this miRNA by overexpression or knockdown seem to mostly affect the $\beta$-cell $[2,22]$. The expression of genes regulated by miR-375 are involved in multiple biological pathways within the $\beta$-cell. These include $\beta$ cell proliferation and differentiation, transcription factor regulation, insulin biosynthesis, insulin granule exocytosis and modulation of ion channels [22]. The expression level of miR-375 is relatively stable, and the miRNA has been used as endogenous control in at least one study [23]. The levels of miR-375 are not differentially-expressed in islets from human T2D individuals as compared to nondiabetic (ND) controls $[24,25]$ and there are little changes in blood/serum miR-375 in T2D [26]. This is most likely explained by the high abundance and stability of miR-375 in islets and the broad target network influencing many functions. In view of the abundance of miR-375 in $\beta$-cells this miRNA has been proposed as a biomarker of $\beta$-cell loss in Type 1 diabetes [27]. However, the analysis of sera from newly diagnosed T1D patients led to contradictory results $[28,29]$.

Other highly abundant miRNAs in islet cells are miR-29a and miR-29b. These miRNAs cause constitutive repression of a monocarboxylase transporter (MCT-1; SLC16A1)[23]. MCT-1 is one of the "disallowed/forbidden" proteins in the $\beta$-cells and its inhibition ensures that only glucose-derived rather than exogenous pyruvate is metabolized by the mitochondria. Suppression of MCT-1 prevents muscle-derived pyruvate from stimulating insulin release during exercise [30].

Also, miR-200 family and miR-7 are highly expressed in islet cells. Members of the miR-200 family were demonstrated to influence $\beta$-cell survival and ablation of several of them in mice protects $\beta$-cells 
against apoptosis [31]. Studies in mice revealed that miR-7a regulates $\beta$-cell function by targeting genes controlling insulin granule exocytosis [32]. MiR-9, one of the first detected islet miRNAs, also regulates exocytosis of insulin granules. Here increased levels of the miRNA repress the transcription factor Onecut-2 and increase the expression of granuphilin [1], a negative regulator of insulin exocytosis.

Many miRNAs influence the process of insulin gene transcription and insulin biosynthesis. Knock-down of a set of miRNAs, including miR-24, miR-26, miR-182, and miR-148 in isolated islets and cultured $\beta$ cells reduced insulin promoter activity and, consequently, insulin content [33]. As mentioned above, the islet abundant miR-375 was also reported to influence insulin production through 3phosphoinositide dependent protein kinase-1 (Pdpk1) [34, 35], although the published data are contradictory. However, this discrepancy may be explained by the different dosage and cell-types used in the experiments. In another study, miR-204 was demonstrated to regulate the expression of MafA [36], a key transcription factor controlling insulin expression. MafA expression is also indirectly influenced by miR-132 and overexpression of this miRNA in rat islets increases the level of this transcription factor [37].

In all, miRNAs are involved in a variety of processes in the $\beta$-cell. They regulate pathways important for development, proliferation and differentiation and other processes forming the $\beta$-cell identity. MiRNAs also control many key $\beta$-cell functions including insulin production, transport and exocytosis of insulin granules, cellular metabolism and the expression of specific ion-channels. Thus, all these processes can be affected by changes in miRNA expression.

\section{Genetic and epigenetic regulation of miRNAs}

The pri-miRNAs are transcribed together with the host-gene or through their own promoter. There are relatively few studies that have investigated the transcriptional regulation of miRNAs in islet cells. Two regulatory elements have been described nearby the miR-375 precursor gene located in an intergenic region on chromosome 2 [38]. The first domain relates to the proximal promoter, whereas the second may correspond to a distal enhancer. Keller et al. further showed that the transcription factor NeuroD1 cooperates with Pdx1 to regulate the transcription of miR-375 [38]. The two miRNAs miR-132 and miR212 are generated from the same transcript and form a miRNA cluster. These two miRNAs share also the same seed sequence and thus belong to the same family. In humans, the gene transcribing the miR-132/miR-212 cluster is located in an intergenic region on chromosome 17p13.3. Transcriptional regulation of this miRNA cluster in $\beta$-cells includes the novel transcription factor Calmodulin Binding Transcription Activator 1 (CAMTA1) [39] and the cAMP responsive element binding protein (CREB) together with the co-activator CAMP-regulated transcriptional co-activator 1 (CRTC1) [40]. Interestingly, the expression of CAMTA1 is reduced in islets from the GK-rat [39].

There are studies showing that expression and/or function of miRNAs can be modulated by other noncoding RNAs. Indeed, the long non-coding RNA (IncRNA) ANRIL was reported to regulate miRNA networks [41]. The gene ANRIL is present in the CDKN2A/B genomic locus and variations at this gene locus are linked to increased risk of T2D. ANRIL binds directly to the miRNAs acting as a miRNA "sponge" or through epigenetic regulation of miRNA transcription. Two conserved miRNAs, miR-675$3 p$ and miR-675-5p, are directly generated from one of the exons of the IncRNA H19. These two miRNAs are expressed at very low level in adult islets and there is so far no evidence for a role in the control of fully mature $\beta$-cell functions [42]. However, H19 may affect $\beta$-cell expansion through repression of another miRNA, let-7 [42]. There is also emerging evidence indicating that circular RNAs (circRNAs), another recently discovered class of non-coding RNAs [43] modulate the activity of miRNAs. 
Thus, the circRNAs ciRS7 and circHIPK3 were reported to sequester miR-7 and other islet miRNAs and to contribute to the regulation of $\beta$-cell functions $[44,45]$.

Recent studies in human islets point to epigenetic regulation of gene expression as a factor contributing to impaired insulin secretion and to the development of T2D [46]. Studies in human islets showed high methylation of the DLK1-MEG3 cluster in T2D islets [21]. In addition, it was discovered that this region contains cell-specific histone modifications and a large number of miRNA genes. In all, the DLK1-MEG3 cluster contains 54 miRNAs of which miR-376 and miR-432 were demonstrated to control islet amyloid polypeptide (IAPP) level, which is involved in $\beta$-cell apoptosis. Epigenetic regulation of miRNA expression was further demonstrated in a study investigating epigenetic differences between sexes in human islets. Differential methylation between sexes was associated with differences in the expression of miR-660 and miR-532 and related target genes [47].

The largest proportion of genetic variations associated with T2D is found in the islet of Langerhans [48]. Potentially, genetic variants within miRNA genes or their regulatory elements could result in differential expression of these small RNAs, favoring the development of T2D. However, there are currently only very few documented genetic variations at miRNA gene sites associated with T2D [49]. One reason for current scarce knowledge regarding the genetic regulation of miRNA expression are coupled to the difficulty of precisely mapping promoters and transcription starting sites for miRNA genes. This is a rather complex work and annotations are far from precise in this area, because miRNAs are generated from long primary transcripts that are rapidly cleaved by Drosha. The transcription start sites are therefore located far from the mature miRNA and cannot be easily inferred from the genomic location of the mature miRNA [50].

While many of the changes in miRNA level under physiological or pathological conditions are dictated by regulatory events occurring inside the $\beta$-cells, the miRNA profile can also be influenced by the delivery of miRNAs carried by exosomes released by other cells. Indeed, under insulin resistance conditions, exosomes released by skeletal muscles were shown to allow the delivery of miR-16 to $\beta$ cells and to favour the expansion of the insulin-secreting cells [51]. On the other hand, the exosomes of lymphocytes invading the islets during the initial phases of Type 1 diabetes were found to transfer a subset of miRNAs, contributing to $\beta$-cell apoptosis [52]. An analogous mechanism may permit the exchange of miRNAs between $\beta$-cells and other cells present within the islets or with other metabolic tissues $[53,54]$.

\section{Environmental factors affecting miRNA expression}

Low physical activity and obesity are environmental risk factors for the development of T2D. Obesity is associated with larger amounts of ectopic fat, and also the $\beta$-cells in human islets contain more fatdroplets with increased BMI of the donor [55]. Both the rise in plasma levels of glucose and lipids affect $\beta$-cell function and the expression of many genes. A summary of some of the miRNAs regulated by glucose and lipids are presented in Table 1.

Glucose is a positive stimulator of insulin secretion in the short term ( $\sim \mathrm{h})$, whereas long-term incubation of islets with high levels of glucose leads to glucotoxicity. In studies performed in vitro, 24$72 \mathrm{~h}$ is enough to detect glucotoxicity. The levels of several miRNAs are glucose-dependent including miR-375, miR-212/miR-132, and miR-130a [34, 56]. Incubation of Wistar rat islets for $1 \mathrm{~h}$ in increasing glucose concentrations raises the levels of miR-212 and reduces those of miR-130a [56]. As elevated levels of miR-130a diminish intracellular ATP [24], reduced expression of this miRNA might increase ATP during short term incubations, which is essential for enhanced insulin secretion. 
More miRNAs are differentially-expressed after 24h incubation of Wistar rat islets in high glucose [56], showing that miRNAs may take part in $\beta$-cell adjustments under glucotoxic conditions. As after $1 \mathrm{~h}$ incubation, levels of miR-212/miR-132 increase while miR-130a expression decrease. Interestingly, GK-rat islets (that are exposed to high blood glucose for an even longer period) display not only increased expression of miR-212/miR-132 but also higher levels of miR-130a. Thus, these observations suggest that the expression of miR-212/miR-132 is elevated due to increased glucose. The reasons of elevated levels of miR-130a in the islets of GK rats is hard to determine. They may be due to the genetic background of the GK-rat islets or to the fact that 24-h incubation in vitro is too short to mimic the glucotoxic effects occurring in vivo. Indeed, along this line, miR-130a was found to be elevated also in islets from human T2D donors [24].

Glucose also indirectly influence the expression of some miRNAs. E.g. glucose-mediated regulation of thioredoxin-interacting protein (TXNIP) increases miR-204 expression [36] and glucose-regulated changes in AMPK activity reduce the levels of miR-184 [57]. Other changes in miRNA expression associated with glucose occur during weaning [58]. Experiments performed on rats show that when pups go from a fat-rich milk diet to a carbohydrate rich diet, the expression of $>60$ miRNAs is modified. Many of these changes are prevented when maintaining the pups on a fat-rich diet, suggesting that these miRNAs are essential in achieving optimal glucose-regulated insulin secretion. One of the clusters showing reduced expression after weaning is miR-17/miR-92. miR-17 targets Phosphofructokinase (Pfkp) and phosphatase with tensin homology (Pten) and knockdown of miR-17 in an insulin-secreting cell line increases insulin secretion.

Interestingly some of the miRNAs regulated by glucose are also differentially expressed after treatment of islets with palmitate to mimic lipotoxicity. These include miR-132/miR-212 and miR-184. Nesca et al showed that miR-132 expression is elevated by incubation with palmitate for 48 and $72 \mathrm{~h}$, whereas miR-184 expression decreases after the same treatment [37]. These miRNAs are also differentially expressed in mice given a high fat diet (HFD).

\section{Changes in islet miRNA levels associated with diabetes}

Several studies have investigated the changes in islet miRNA expression occurring in T2D models (Table 1). Zhao et al. carried out a systematic analysis of the islet miRNA profile of obese ob/ob mice in diabetes resistant (B6) and diabetes prone (BTBR) backgrounds [59]. They identified several miRNAs that are differentially expressed between the two mice strains and are responsive to obesity. In particular, they observed a very strong decrease in the expression of miR-184 and the up-regulation of miR-132 and miR-212 in response to obesity [59]. They also found changes in the level of miR-34a, miR-146a and miR-152. Modifications in the expression of these and many other miRNAs, including miR-204 and miR-200 family members, were also detected in a global profiling of pancreatic islets from $o b / o b$ mice $[31,59,60]$, in prediabetic and diabetic $d b / d b$ mice, lacking the leptin receptor [37], and in mice kept on a high fat diet [37]. Many of these changes are likely to be directly linked to obesity and to elevated free fatty acid levels. However, the up-regulation of miR-132, miR-212 and probably others may also be caused by prolonged hyperglycemia since it can be mimicked in vitro by exposing the islets to high glucose concentrations $[37,56]$ and is also observed in the islets of GK rats, a model of T2D that is not associated with obesity [56].

The changes in islet miRNA expression detected under diabetic conditions may directly contribute to the development of the disease. However, some of them may be the consequence of the exposure of the cells to chronic hyperglycemia and hyperlipidemia and can even have positive impacts on $\beta$-cell functions. Indeed, while the alterations in the level of several miRNAs observed in the islets of diabetic animals have deleterious effects on $\beta$-cell activities [24, 31, 36, 37], the down-regulation of miR-184 
promotes $\beta$-cell proliferation both in vitro and in vivo $[37,60]$. Similar effects were also elicited by the up-regulation of miR-132 and were accompanied by an improvement in the secretory activity of pancreatic $\beta$-cells [37]. Thus, the changes in the expression of miR-184 and miR-132 are most probably part of an adaptive program allowing $\beta$-cells to release more insulin and to compensate for the diminished sensitivity of the target tissues. Another miRNA that appears to be involved in the adaptation of $\beta$-cells to insulin resistance is miR-7a. This miRNA is highly abundant in $\beta$-cells and regulates the expression of several key components necessary for insulin exocytosis [32]. The level of miR-7a in pancreatic islets is reduced under moderate insulin resistance conditions, resulting in improved insulin secretion. However, the expression of miR-7a raises progressively under severe diabetic conditions and can reach levels even higher than in healthy individuals, probably contributing to defective insulin release and to the progression of the disease [32].

At present, only few studies investigated the potential changes in miRNA levels occurring in the islets of T2D patients. As mentioned above, Kameswaran et al. identified a cluster of miRNAs in the DLK1MEG3 imprinted locus on chromosome $14 q 32$ that is specifically expressed in human $\beta$-cells and is strongly downregulated in the islets of T2D organ donors [21]. The reduction of the level of these miRNAs was correlated to epigenetic changes and to the hypermethylation of the promoter sequences. Interestingly, the miRNAs belonging to the DLK1-MEG3 cluster were found to control the expression of genes involved in $\beta$-cell survival [21], suggesting that reduced levels of these miRNAs may contribute to T2D pathogenesis.

Locke et al. used TaqMan arrays to perform a global profiling of islet miRNAs in cohorts of individuals with and without T2D [61]. These authors did not observe significant differences in the level of the miRNAs generated from the DLK1-MEG3 cluster. However, they detected in the islets obtained from T2D donors a dramatic increase of miR-187, a miRNA that was also very strongly up-regulated in the analysis carried out by Kameswaran et al. [21]. Interestingly, the level of miR-187 in human islets was found to be inversely correlated to insulin secretion. Moreover, overexpression of miR-187 in rat islets resulted in a drastic reduction of glucose-induced insulin release [61]. The mechanisms underlying the effect of miR-187 on insulin secretion remain to be precisely defined. However, part of the action may be exerted by modulating the expression of HIPK3 (homeodomain-interacting protein kinase 3), a protein kinase which is required for normal insulin secretion [62] and is a direct target of this miRNA [61].

Few other studies reported changes in the level of specific miRNAs in the islets of T2D patients. Bomelson et al. investigated the expression of 8 miRNAs (miR-21, miR-122, miR-127-3p, miR-184, miR195, miR-375, miR-492 and miR-493*) in the islets of healthy and glucose intolerant individuals [63]. They found a significant increase in miR-21 in the islets of glucose-intolerant individuals and a tendency for a rise of miR-127-3p and miR-375. In contrast, Sebastiani et al. assessed the expression of miR-124a and observed higher levels of this miRNA in the islets of T2D patients but no significant differences in the expression of miR-375 [25]. Tattitoka et al. reported a decrease in miR-184 expression in the islets of T2D patients, reminiscent of the changes observed by different groups in animal models of T2D [60]. Finally, Ofori et al reported elevated expression of miR-130a, miR-130b and miR-152 in human islets of T2D patients [24]. Expression of these three miRNAs in human islets was highly correlated. Elevation of the expression of each of these miRNAs reduced the level of the common target Pyruvate Dehydrogenase E1 Alpha 1 Subunit (PDHA1), thereby reducing intracellular ATP and insulin secretion.

As described above, the analysis of the islet miRnome revealed changes in many miRNAs occurring in conjunction with T2D development. However, only few of them were consistently observed. In animal 
models, this is most probably explained by differences in the severity and in the progression of the disease. In fact, the level of some miRNAs is already modified in response to moderate insulin resistance while other miRNAs display altered expression only under severe pathophysiological conditions. Moreover, miR-7a and miR-200 family members show even opposite expression changes during the compensatory phases preceding the appearance of diabetes and after the disease is fully established, potentially leading to contradictory conclusions. Experiments performed with islets of human T2D donors are facing obstacles that are even more difficult to overcome. In fact, in contrast to rodent models, the number of available samples is usually very limited and, consequently, many confounding factors including the genetic background of the donors, the type of treatment, the quality of the glycemic control, the duration of the disease etc. cannot be controlled, inevitably resulting in a high variability of the data. Added to this, the analysis of the miRnome struggle with technical variabilities between laboratories such as the use of different platforms and strategies for normalization of derived data. Currently, islet gene expression platforms are set-up to try to merge results from different islet preparations (e.g. TIGER data portal, https://www.t2dsystems.eu/tigerdatabase). In the near future, also miRNA expression data should be added to such a system.

\section{miRNAs as potential therapeutic targets for T2D}

As described above, T2D is associated with numerous changes in the islet miRNA profile. Thus, strategies permitting to correct the level of selected miRNAs could potentially allow to prevent or even treat the disease. Indeed, knockout mice lacking miR-200 family members were found to be resistant to streptozotocin-induced diabetes [31]. Moreover, the absence of this family of miRNAs permitted to prevent the development of a severe form of diabetes in Akita mice [31]. It is an exciting time for miRNA therapeutics and several miRNA drug candidates are in phase 1 and phase 2 clinical trials. This include e.g. a phase 1 study with a Looked Nucleic Acid (LNA) based drug to inhibit miR-92 for future potential in wound healing and a phase 2 trial for miR-155 for patients with a specific form of T-cell lymphoma [64]. However, so far only very few studies attempted to treat T2D in animal models using strategies that could be potentially translated to humans. Song et al. observed a decrease in miR-26a level in the islets of $d b / d b$ mice and of mice kept on a high fat diet for 6 months [65]. They found that the injection of an adeno-associated virus (AAV8) construct allowing the overexpression of this miRNA is sufficient to prevent the rise in fasting blood glucose levels normally observed in high-fat diet fed mice [65]. This effect was linked to improved $\beta$-cell function and was attributed to the reduction of the expression of PTEN. Unfortunately, the manuscript does not include any description of the AAV8 construct and it is unclear whether the expression of the miRNA was driven by a $\beta$-cell specific promoter.

Mulder et al. used a different approach to treat high-fat diet fed mice. In this case, the authors generated an AAV8 construct that enables the overexpression of miR-132 [66], a miRNA with a beneficial impact on $\beta$-cell function and that is normally involved in the adaptation of $\beta$-cells to insulin resistance (see above). The expression of the construct was under the control of the rat insulin promoter and was therefore restricted to $\beta$-cells [66]. Interestingly, overexpression of miR-132 did not affect glucose homeostasis in mice kept on regular chow diet but enhanced insulin secretion and increased $\beta$-cell proliferation in mice on high-fat diet, resulting in improved blood glucose control [66]. In a more recent study, Bijirk et al. investigated the effect of in vivo injection of cholesterol-conjugated antagomirs targeting miR-132 on islet function in mice [67]. It was verified that miR-132 expression was reduced in the islets, and the treatment resulted in a rise in insulin release and a decrease in blood glucose levels. The discrepancy with previous studies might be due to dose and species differences or to effects exerted on other tissues that indirectly affect the activity of $\beta$-cells. However, this study 
provides a proof of concept that antagomirs might hold a therapeutic potential, especially if coupled with targeted Looked Nucleic Acid (LNA) molecules, which are more stable in blood [68].

\section{Concluding remarks and future perspectives}

During the last years, we have gathered large amount of information about the contribution of miRNAs in the control of $\beta$-cell function and about their involvement in the development of T2D. In the coming years, we will be able to capitalize on this knowledge to design innovative strategies for preventing and treating the disease. We now know that key miRNAs regulate the secretory activity of $\beta$-cells and/or control the $\beta$-cell mass (Figure 1). Genetic and environmental factors involved in the pathogenesis of T2D influence the expression of these miRNAs. These changes can lead either to dysfunctional insulin secretion, as is the case for miR-130a/b and miR-152, or in compensatory adaptations in $\beta$-cell activities, as is the case for miR-184 and miR-212/miR-132. In view of this, it is possible to envisage both therapeutic strategies aiming at correcting the level of the miRNAs displaying a negative impact on $\beta$-cells or approaches promoting miRNA changes that facilitate the adaptation of $\beta$-cells to increased insulin demand. However, for diabetes treatment we urgently need better tools to target one or more miRNAs specifically in $\beta$-cells. Indeed, none of the miRNAs studied so far is exclusively expressed in insulin-secreting cells and without a precise delivery of the miRNA-targeting tools to $\beta$-cells, any of these therapeutic strategies will most likely cause unacceptable side effects.

Another aspect concerning the miRNAs that is rapidly expanding and taking increasing importance is their use as biomarkers. Indeed, measurements of miRNAs in plasma/serum have proved to be useful tools for predicting the manifestation of T2D or its associated complications $[5,69]$. In view of the exiguous number of $\beta$-cells, changes in their miRNA profile are unlikely to be reflected in sizeable modifications in plasma miRNA levels. However, in the future this obstacle may be overcome by approaches permitting the selective isolation from blood samples of vesicles released by $\beta$-cells [70].

Despite rapid progress, we are only beginning to appreciate the contribution of miRNAs to islet function and to T2D development. For a full understanding of their role in T2D, future work will necessitate building of large-scale miRNA-mRNA networks and a better definition of genetic and epigenetic miRNA interactions. Moreover, although so far research in this field has almost exclusively focused on $\beta$-cells, miRNAs are very likely to regulate the function of other islet cells [71] and may thus contribute to T2D through other, yet to be identified, mechanisms. This promises new exciting discoveries also for the coming years.

\section{Acknowledgement}

We thank colleagues and friends for fruitful discussions in this area. Work by LE in this area is supported by grants from the Swedish Foundation for Strategic Research (IRC-LUDC), the Swedish Research Council (Project grant to LE, SFO-EXODIAB), Region Skåne-ALF, The Swedish Diabetes Foundation, the Diabetes Wellness Network Sweden. RR is supported by the Swiss National Science Foundation grant 310030-169480.

\section{References}

[1] Plaisance V, Abderrahmani A, Perret-Menoud V, Jacquemin P, Lemaigre F, Regazzi R. MicroRNA-9 controls the expression of Granuphilin/SIp4 and the secretory response of insulin-producing cells. The Journal of biological chemistry. 2006;281:26932-42. 
[2] Poy MN, Eliasson L, Krutzfeldt J, Kuwajima S, Ma X, Macdonald PE, et al. A pancreatic islet-specific microRNA regulates insulin secretion. Nature. 2004;432:226-30.

[3] Esguerra JL, Nagao M, Ofori JK, Wendt A, Eliasson L. MicroRNAs in islet hormone secretion. Diabetes Obes Metab. 2018;in press.

[4] LaPierre MP, Stoffel M. MicroRNAs as stress regulators in pancreatic beta cells and diabetes. Mol Metab. 2017;6:1010-23.

[5] Guay C, Regazzi R. Circulating microRNAs as novel biomarkers for diabetes mellitus. Nat Rev Endocrinol. 2013;9:513-21.

[6] Halban PA, Polonsky KS, Bowden DW, Hawkins MA, Ling C, Mather KJ, et al. beta-cell failure in type 2 diabetes: postulated mechanisms and prospects for prevention and treatment. Diabetes Care. 2014;37:1751-8.

[7] Rorsman P, Ashcroft FM. Pancreatic beta-Cell Electrical Activity and Insulin Secretion: Of Mice and Men. Physiol Rev. 2018;98:117-214.

[8] Hall E, Jonsson J, Ofori JK, Volkov P, Perfilyev A, Dekker-Nitert M, et al. Glucolipotoxicity Alters Insulin Secretion via Epigenetic Changes in Human Islets. Diabetes. 2019.

[9] Olofsson CS, Collins S, Bengtsson M, Eliasson L, Salehi A, Shimomura K, et al. Long-term exposure to glucose and lipids inhibits glucose-induced insulin secretion downstream of granule fusion with plasma membrane. Diabetes. 2007;56:1888-97.

[10] Bartel DP. Metazoan MicroRNAs. Cell. 2018;173:20-51.

[11] Lee $\mathrm{Y}$, Jeon K, Lee JT, Kim S, Kim VN. MicroRNA maturation: stepwise processing and subcellular localization. Embo J. 2002;21:4663-70.

[12] Bartel DP. MicroRNAs: target recognition and regulatory functions. Cell. 2009;136:215-33.

[13] Gregory RI, Yan KP, Amuthan G, Chendrimada T, Doratotaj B, Cooch N, et al. The Microprocessor complex mediates the genesis of microRNAs. Nature. 2004;432:235-40.

[14] Yi R, Doehle BP, Qin Y, Macara IG, Cullen BR. Overexpression of exportin 5 enhances RNA interference mediated by short hairpin RNAs and microRNAs. RNA. 2005;11:220-6.

[15] Kalis M, Bolmeson C, Esguerra JL, Gupta S, Edlund A, Tormo-Badia N, et al. Beta-cell specific deletion of Dicer1 leads to defective insulin secretion and diabetes mellitus. PLoS One. 2011;6:e29166.

[16] Zhang K, Zhang X, Cai Z, Zhou J, Cao R, Zhao Y, et al. A novel class of microRNA-recognition elements that function only within open reading frames. Nat Struct Mol Biol. 2018;25:1019-27.

[17] Chipman LB, Pasquinelli AE. miRNA Targeting: Growing beyond the Seed. Trends Genet. 2019;35:215-22.

[18] Wendt A, Esguerra JL, Eliasson L. Islet microRNAs in health and type-2 diabetes. Curr Opin Pharmacol. 2018;43:46-52.

[19] Bravo-Egana V, Rosero S, Molano RD, Pileggi A, Ricordi C, Dominguez-Bendala J, et al. Quantitative differential expression analysis reveals miR-7 as major islet microRNA. Biochemical and biophysical research communications. 2008;366:922-6.

[20] van de Bunt M, Gaulton KJ, Parts L, Moran I, Johnson PR, Lindgren CM, et al. The miRNA Profile of Human Pancreatic Islets and Beta-Cells and Relationship to Type 2 Diabetes Pathogenesis. PLOS ONE. 2013;8:e55272.

[21] Kameswaran V, Bramswig NC, McKenna LB, Penn M, Schug J, Hand NJ, et al. Epigenetic regulation of the DLK1-MEG3 microRNA cluster in human type 2 diabetic islets. Cell Metab. 2014;19:135-45.

[22] Eliasson L. The small RNA miR-375 - a pancreatic islet abundant miRNA with multiple roles in endocrine beta cell function. Mol Cell Endocrinol. 2017;456:95-101.

[23] Pullen TJ, da Silva Xavier G, Kelsey G, Rutter GA. miR-29a and miR-29b contribute to pancreatic beta-cell-specific silencing of monocarboxylate transporter 1 (Mct1). Mol Cell Biol. 2011;31:3182-94.

[24] Ofori JK, Salunkhe VA, Bagge A, Vishnu N, Nagao M, Mulder H, et al. Elevated miR130a/miR130b/miR-152 expression reduces intracellular ATP levels in the pancreatic beta cell. Sci Rep. 2017;7:44986.

[25] Sebastiani G, Po A, Miele E, Ventriglia G, Ceccarelli E, Bugliani M, et al. MicroRNA-124a is hyperexpressed in type 2 diabetic human pancreatic islets and negatively regulates insulin secretion. Acta Diabetol. 2015;52:523-30. 
[26] Latreille M, Herrmanns K, Renwick N, Tuschl T, Malecki MT, McCarthy MI, et al. miR-375 gene dosage in pancreatic beta-cells: implications for regulation of beta-cell mass and biomarker development. Journal of molecular medicine. 2015;93:1159-69.

[27] Erener S, Mojibian M, Fox JK, Denroche HC, Kieffer TJ. Circulating miR-375 as a biomarker of betacell death and diabetes in mice. Endocrinology. 2013;154:603-8.

[28] Erener S, Marwaha A, Tan R, Panagiotopoulos C, Kieffer TJ. Profiling of circulating microRNAs in children with recent onset of type 1 diabetes. JCI Insight. 2017;2:e89656.

[29] Marchand L, Jalabert A, Meugnier E, Van den Hende K, Fabien N, Nicolino M, et al. miRNA-375 a Sensor of Glucotoxicity Is Altered in the Serum of Children with Newly Diagnosed Type 1 Diabetes. J Diabetes Res. 2016;2016:1869082.

[30] Ishihara $\mathrm{H}$, Wang $\mathrm{H}$, Drewes $\mathrm{LR}$, Wollheim CB. Overexpression of monocarboxylate transporter and lactate dehydrogenase alters insulin secretory responses to pyruvate and lactate in beta cells. J Clin Invest. 1999;104:1621-9.

[31] Belgardt BF, Ahmed K, Spranger M, Latreille M, Denzler R, Kondratiuk N, et al. The microRNA-200 family regulates pancreatic beta cell survival in type 2 diabetes. Nature medicine. 2015;21:619-27.

[32] Latreille M, Hausser J, Stutzer I, Zhang Q, Hastoy B, Gargani S, et al. MicroRNA-7a regulates pancreatic beta cell function. J Clin Invest. 2014;124:2722-35.

[33] Melkman-Zehavi T, Oren R, Kredo-Russo S, Shapira T, Mandelbaum AD, Rivkin N, et al. miRNAs control insulin content in pancreatic beta-cells via downregulation of transcriptional repressors. Embo J. 2011;30:835-45.

[34] El Ouaamari A, Baroukh N, Martens GA, Lebrun P, Pipeleers D, van Obberghen E. miR-375 targets 3 '-phosphoinositide-dependent protein kinase-1 and regulates glucose-induced biological responses in pancreatic beta-cells. Diabetes. 2008;57:2708-17.

[35] Nathan G, Kredo-Russo S, Geiger T, Lenz A, Kaspi H, Hornstein E, et al. MiR-375 promotes redifferentiation of adult human beta cells expanded in vitro. PLoS One. 2015;10:e0122108.

[36] Xu G, Chen J, Jing G, Shalev A. Thioredoxin-interacting protein regulates insulin transcription through microRNA-204. Nature medicine. 2013;19:1141-6.

[37] Nesca V, Guay C, Jacovetti C, Menoud V, Peyot ML, Laybutt DR, et al. Identification of particular groups of microRNAs that positively or negatively impact on beta cell function in obese models of type 2 diabetes. Diabetologia. 2013;56:2203-12.

[38] Keller DM, McWeeney S, Arsenlis A, Drouin J, Wright CV, Wang H, et al. Characterization of pancreatic transcription factor Pdx-1 binding sites using promoter microarray and serial analysis of chromatin occupancy. J Biol Chem. 2007;282:32084-92.

[39] Mollet IG, Malm HA, Wendt A, Orho-Melander M, Eliasson L. CAMTA1-Calcium-Calmodulin Transcriptional Activator 1 , a new player in the regulation of microRNAs and insulin secretion. Diabetologia. 2015;58:S88-S.

[40] Malm HA, Mollet IG, Berggreen C, Orho-Melander M, Esguerra JL, Goransson O, et al. Transcriptional regulation of the miR-212/miR-132 cluster in insulin-secreting beta-cells by cAMPregulated transcriptional co-activator 1 and salt-inducible kinases. Mol Cell Endocrinol. 2016;424:2333.

[41] Kong Y, Hsieh CH, Alonso LC. ANRIL: A IncRNA at the CDKN2A/B Locus With Roles in Cancer and Metabolic Disease. Front Endocrinol (Lausanne). 2018;9:405.

[42] Sanchez-Parra C, Jacovetti C, Dumortier O, Lee K, Peyot ML, Guay C, et al. Contribution of the Long Noncoding RNA H19 to beta-Cell Mass Expansion in Neonatal and Adult Rodents. Diabetes. 2018;67:2254-67.

[43] Kristensen LS, Andersen MS, Stagsted LVW, Ebbesen KK, Hansen TB, Kjems J. The biogenesis, biology and characterization of circular RNAs. Nat Rev Genet. 2019.

[44] Stoll L, Sobel J, Rodriguez-Trejo A, Guay C, Lee K, Veno MT, et al. Circular RNAs as novel regulators of beta-cell functions in normal and disease conditions. Mol Metab. 2018;9:69-83.

[45] Xu H, Guo S, Li W, Yu P. The circular RNA Cdr1as, via miR-7 and its targets, regulates insulin transcription and secretion in islet cells. Sci Rep. 2015;5:12453.

[46] Ling C, Ronn T. Epigenetics in Human Obesity and Type 2 Diabetes. Cell Metab. 2019;29:1028-44. 
[47] Hall E, Volkov P, Dayeh T, Esguerra JL, Salo S, Eliasson L, et al. Sex differences in the genome-wide DNA methylation pattern and impact on gene expression, microRNA levels and insulin secretion in human pancreatic islets. Genome Biol. 2014;15:522.

[48] van de Bunt M, Gloyn AL. From genetic association to molecular mechanism. Curr Diab Rep. 2010;10:452-66.

[49] Locke JM, Lango Allen H, Harries LW. A rare SNP in pre-miR-34a is associated with increased levels of miR-34a in pancreatic beta cells. Acta Diabetol. 2014;51:325-9.

[50] Marsico A, Huska MR, Lasserre J, Hu H, Vucicevic D, Musahl A, et al. PROmiRNA: a new miRNA promoter recognition method uncovers the complex regulation of intronic miRNAs. Genome Biol. 2013;14:R84.

[51] Jalabert A, Vial G, Guay C, Wiklander OP, Nordin JZ, Aswad H, et al. Exosome-like vesicles released from lipid-induced insulin-resistant muscles modulate gene expression and proliferation of beta recipient cells in mice. Diabetologia. 2016;59:1049-58.

[52] Guay C, Kruit JK, Rome S, Menoud V, Mulder NL, Jurdzinski A, et al. Lymphocyte-Derived Exosomal MicroRNAs Promote Pancreatic beta Cell Death and May Contribute to Type 1 Diabetes Development. Cell Metab. 2019;29:348-61 e6.

[53] Guay C, Regazzi R. Exosomes as new players in metabolic organ cross-talk. Diabetes Obes Metab. 2017; 19 Suppl 1:137-46.

[54] Mori MA, Ludwig RG, Garcia-Martin R, Brandao BB, Kahn CR. Extracellular miRNAs: From Biomarkers to Mediators of Physiology and Disease. Cell Metab. 2019.

[55] Rosengren AH, Braun M, Mahdi T, Andersson SA, Travers ME, Shigeto M, et al. Reduced insulin exocytosis in human pancreatic beta-cells with gene variants linked to type 2 diabetes. Diabetes. 2012;61:1726-33.

[56] Esguerra JL, Bolmeson C, Cilio CM, Eliasson L. Differential glucose-regulation of microRNAs in pancreatic islets of non-obese type 2 diabetes model Goto-Kakizaki rat. PLoS One. 2011;6:e18613.

[57] Martinez-Sanchez A, Nguyen-Tu MS, Cebola I, Yavari A, Marchetti P, Piemonti L, et al. MiR-184 expression is regulated by AMPK in pancreatic islets. FASEB J. 2018;32:2587-600.

[58] Jacovetti C, Abderrahmani A, Parnaud G, Jonas JC, Peyot ML, Cornu M, et al. MicroRNAs contribute to compensatory beta cell expansion during pregnancy and obesity. J Clin Invest. 2012;122:3541-51.

[59] Zhao E, Keller MP, Rabaglia ME, Oler AT, Stapleton DS, Schueler KL, et al. Obesity and genetics regulate microRNAs in islets, liver, and adipose of diabetic mice. Mamm Genome. 2009;20:476-85.

[60] Tattikota SG, Rathjen T, McAnulty SJ, Wessels HH, Akerman I, van de Bunt M, et al. Argonaute2 mediates compensatory expansion of the pancreatic beta cell. Cell Metab. 2014;19:122-34.

[61] Locke JM, da Silva Xavier G, Dawe HR, Rutter GA, Harries LW. Increased expression of miR-187 in human islets from individuals with type 2 diabetes is associated with reduced glucose-stimulated insulin secretion. Diabetologia. 2014;57:122-8.

[62] Shojima N, Hara K, Fujita H, Horikoshi M, Takahashi N, Takamoto I, et al. Depletion of homeodomain-interacting protein kinase 3 impairs insulin secretion and glucose tolerance in mice. Diabetologia. 2012;55:3318-30.

[63] Bolmeson C, Esguerra JL, Salehi A, Speidel D, Eliasson L, Cilio CM. Differences in islet-enriched miRNAs in healthy and glucose intolerant human subjects. Biochemical and biophysical research communications. 2011;404:16-22.

[64] Hanna J, Hossain GS, Kocerha J. The Potential for microRNA Therapeutics and Clinical Research. Front Genet. 2019;10:478.

[65] Song Y, Jin D, Jiang X, Lv C, Zhu H. Overexpression of microRNA-26a protects against deficient betacell function via targeting phosphatase with tensin homology in mouse models of type 2 diabetes. Biochemical and biophysical research communications. 2018;495:1312-6.

[66] Mulder NL, Havinga R, Kluiver JL, Groen AK, Kruit JK. AAV8-mediated gene transfer of microRNA132 improves beta-cell function in mice fed a high fat diet. J Endocrinol. 2018.

[67] Bijkerk R, Esguerra JLS, Ellenbroek JH, Au YW, Hanegraaf MAJ, de Koning EJ, et al. In Vivo Silencing of MicroRNA-132 Reduces Blood Glucose and Improves Insulin Secretion. Nucleic Acid Ther. 2019. 
[68] Elmen J, Thonberg $\mathrm{H}$, Ljungberg $\mathrm{K}$, Frieden $\mathrm{M}$, Westergaard $\mathrm{M}, \mathrm{Xu} \mathrm{Y}$, et al. Locked nucleic acid (LNA) mediated improvements in siRNA stability and functionality. Nucleic Acids Res. 2005;33:439-47. [69] Gallo W, Esguerra JLS, Eliasson L, Melander O. miR-483-5p associates with obesity and insulin resistance and independently associates with new onset diabetes mellitus and cardiovascular disease. PLoS One. 2018;13:e0206974.

[70] Vallabhajosyula P, Korutla L, Habertheuer A, Yu M, Rostami S, Yuan CX, et al. Tissue-specific exosome biomarkers for noninvasively monitoring immunologic rejection of transplanted tissue. J Clin Invest. 2017;127:1375-91.

[71] Dalgaard LT, Eliasson L. An 'alpha-beta' of pancreatic islet microribonucleotides. The international journal of biochemistry \& cell biology. 2017;88:208-19. 
Table and Figure legends

\begin{tabular}{|c|c|c|c|c|c|c|c|}
\hline miRNA & $\begin{array}{l}\text { Glucose } \\
\text { Cells/islets }\end{array}$ & $\begin{array}{l}\text { Palmitate } \\
\text { Cells/islets }\end{array}$ & $\begin{array}{l}\text { HFD } \\
\text { mice }\end{array}$ & $\begin{array}{l}\text { ob/ob } \\
\text { mice }\end{array}$ & $\begin{array}{l}\mathrm{db} / \mathrm{db} \\
\text { mice }\end{array}$ & $\begin{array}{l}\text { GK } \\
\text { rat }\end{array}$ & $\begin{array}{l}\text { Human } \\
\text { T2D islets }\end{array}$ \\
\hline miR-7a/miR-7b & & & $\downarrow$ & $\downarrow$ & $\uparrow$ (adult) & $\uparrow(m i R-7 b)$ & $\downarrow$ \\
\hline miR-130a/miR-130b & $\downarrow$ & & & & & $\uparrow$ & $\uparrow$ \\
\hline miR-132/miR-212 & 个 & 个 & 个 & $\uparrow$ & $\uparrow$ & $\uparrow$ & 一 \\
\hline miR-338 & & & $\downarrow$ & & $\downarrow$ & 一 & \\
\hline miR-152 & $\downarrow$ & & & 个 & & $\uparrow$ & $\uparrow$ \\
\hline miR-184 & $\downarrow$ & $\downarrow$ & $\downarrow$ & $\downarrow$ & $\downarrow$ & 一 & $\downarrow$ \\
\hline miR-187 & & & & & & 一 & $\uparrow$ \\
\hline miR-200 family & & & $\downarrow$ & 一 & $\uparrow$ & 一 & \\
\hline miR-204 & 个 & & & & & - & \\
\hline miR-375 & $\downarrow /-$ & & $\uparrow$ & $\uparrow$ & & - & - \\
\hline
\end{tabular}

Table 1. Summary of key islet miRNAs differentially expressed in T2D. The table describes changes in islet expression induced by nutrients, in diabetes animal models (HFD-treated mouse, ob/ob-mouse and $\mathrm{db} / \mathrm{db}$-mouse models, and the GK-rat model) and human islets from T2D donors. Data are from references [24, 31, 32, 34, 36, 56-58, 60, 61, 67]. $\uparrow$ : increased expression vs control; $\downarrow$ :decreased expression vs control; -: not differentially expressed vs control. 


\section{Genetic and environmetal factors Increased insulin resistance}

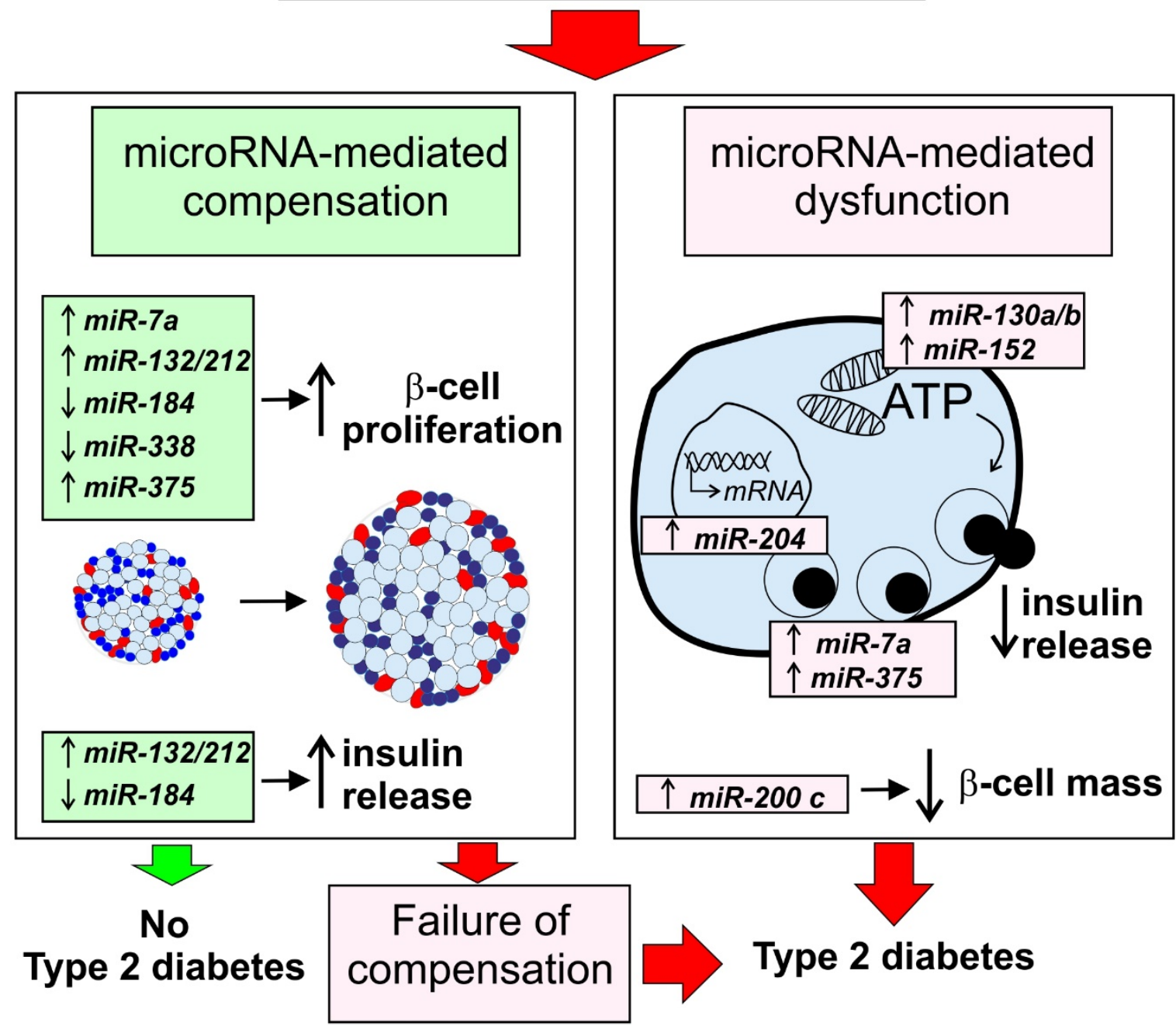

Figure 1. Summary of miRNA regulation of $\beta$-cell function and $\beta$-cell mass during development of T2D. Genetic and environmental factors influence the capacity to secrete enough insulin to cover the organism needs. Increased demand on the $\beta$-cell due to insulin resistance requires augmented secretion from individual cells and enhanced proliferation to enlarge the $\beta$-cell mass. Failure to compensate for the increased demand results in T2D development. The image describes how genetic and/or environmental factors (e.g. increased amount of nutrients) regulate specific miRNAs. Whereas changes in the expression of some miRNAs are part of the aetiology of T2D, others occur as a compensatory mechanism for insulin resistance. Of the miRNAs listed in the image a majority controls both the function and the mass of $\beta$-cells. miR-7a, miR-130a/b, miR-152 and miR-184 are differentially expressed in islets of healthy and T2D donors. Data in this image are from $[24,31,32,34,36,56-58$, $60,61,67]$. 